\title{
Divergence of Fourier series: Corrigenda
}

\section{Masako Izumi and Shin-ichi Izumi}

The authors regret that the following corrections need to be made to their paper [1].

$$
\begin{array}{ll}
\text { p. } 293,1.2 & \text { delete the factor } \frac{1}{2} . \\
\text { p. } 294,1.6 & \text { replace the denominator } 2 \pi l \text { by } \pi l . \\
\text { p. } 295,1 .-4 & \text { replace } \frac{1}{\pi n} \text { by } \frac{1}{n} \text { and } R_{n}^{\prime \prime} \text { by } R_{k}^{\prime \prime} . \\
\text { p. } 295,1 .-3 & \text { replace } \frac{1}{\pi n} \text { by } \frac{1}{n} . \\
\text { p. } 296,1.2 & \text { replace } \frac{-1}{2 \pi} \text { by } \frac{-1}{\pi} . \\
\text { p. } 296,1.3 & \text { multiply the sum by } 2 . \\
\text { p. } 296,1.4,5 & \text { replace } \frac{n+1 / 2}{2 \pi} \text { by } \frac{n+1 / 2}{\pi} . \\
\text { p. } 296,1.10-11 & \text { replace these two } 1 \text { ines by } \\
& \frac{1}{n} U_{N-1}^{\prime}=\frac{1 i m}{M+\infty} \frac{n+1 / 2}{n \pi} \int_{d v}^{M} d l,
\end{array}
$$

where the integrand and the limits of the first integration remain the same.

p. $297,1.1,3,5$ multiply the integrals by $\frac{1}{\pi}$.

p. $297,1.10$ replace $\frac{1}{2 \pi k}$ by $\frac{1}{\pi k}$.

p. 297, 1. 11 replace $\frac{1}{4 \pi k}$ by $\frac{1}{2 \pi k}$.

p. $297,1.12,13$ replace $\frac{1}{4 \pi}$ by $\frac{1}{2 \pi}$.

p. $297,1 .-3,-2$ multiply the sums by 2 . 
p. $298,1.10$ replace $\frac{1}{n}$ on the right hand side by $\frac{2}{\pi n}$.

p. $298,1.12,13$ replace $\frac{\pi}{2 n}$ by $\frac{2}{n}$.

p. $298,1 .-3 \quad$ replace $\frac{1}{2 n}$ by $\frac{2}{n}$.

p. 299, 1. 1 replace $\frac{1}{2 n}$ by $\frac{2}{n}$.

\section{Reference}

[1] Masako Izumi and Shin-ichi Lzumi, "Divergence of Fourier series", BulZ. Austral. Math. Soc. 8 (1973), 289-304.

Department of Mathematics, Institute of Advanced Studies, Australian National University, Canberra, ACT. 